COMPUTATIONAL METHODS IN APPLIED MATHEMATICS, Vol. 8 (2008), No. 1, pp. 86-98

(C) 2008 Institute of Mathematics of the National Academy of Sciences of Belarus

\title{
MOROZOV'S DISCREPANCY PRINCIPLE FOR THE TIKHONOV REGULARIZATION OF EXPONENTIALLY ILL-POSED PROBLEMS
}

\author{
S. G. SOLODKY ${ }^{1}$ and A. MOSENTSOVA ${ }^{1}$
}

\begin{abstract}
The problem of approximate solution of severely ill-posed problems given in the form of linear operator equations of the first kind with approximately known right-hand sides was considered. We have studied a strategy for solving this type of problems, which consists in combinating of Morozov's discrepancy principle and a finite-dimensional version of the Tikhonov regularization. It is shown that this combination provides an optimal order of accuracy on source sets.
\end{abstract}

2000 Mathematics Subject Classification: 65R30, 65R20, 45L10.

Keywords: essentially ill-posed problems, Tikhonov regularization, Morozov's discrepancy principle, source set.

\section{Introduction}

The present article is devoted to the problem of approximate solution of a linear ill-posed problem represented as

$$
A x=y,
$$

where instead of $y$ we have an available approximation $y_{\delta},\left\|y-y_{\delta}\right\| \leqslant \delta$, and $A$ is a linear compact injective operator between Hilbert spaces $X$ and $Y$. We denote the inner products in these spaces by $(\cdot, \cdot)$ and the corresponding norms by $\|\cdot\|$. The symbol $\|\cdot\|$ stands also for the standard operator norm. It will always be clear from the context which space or norm is being considered.

Usually, equation (1.1) is called a severely ill-posed problem if its solution $x_{0}=A^{-1} y$ has a finite "smoothness" in some sense, but $A$ is an infinitely smoothing operator. Such problems are exemplified by exponentially ill-posed problems. A distinguishing characteristic of the latter problems is the fact that $x_{0}$ belongs to some subspace $V$, continuously embedded in $X$, and the singular values of the canonical embedding operator $J_{V}$ from $V$ into $X$ tend to zero at a polynomial rate, while the singular values $\left\{\sigma_{l}\right\}_{l=1}^{\infty}$ of the operator $A$ tend to zero quickly enough so that $\underline{c} \exp \left(-\exp \ldots \exp l^{r}\right) \leqslant \sigma_{l} \leqslant \bar{c} \exp \left(-\exp \ldots \exp l^{r}\right)$ for some constants $\bar{c} \geqslant \underline{c}>0$, where the function exp appears $K \geqslant 1$ times. Following [11; 1 , section 2.1] here it is natural to assume that

$$
x_{0} \in M_{p, \rho}^{\log }(A):=\left\{x: x=(\underbrace{\ln \ldots \ln }_{K \text { times }}\left(A^{*} A\right)^{-1})^{-p} v, \quad\|v\| \leqslant \rho\right\}
$$

\footnotetext{
${ }^{1}$ Institute of Mathematics, National Akademy of Sciences, Tereschenkivska Str. 3, 01601 Kiev, Ukraine. E-mail: solodky@imath.kiev.ua, annam@ukr.net
} 
for some $p>p_{0}$ and $\rho>0$, where the operator function $\left(\ln \ldots \ln \left(A^{*} A\right)^{-1}\right)^{-p}$ is well defined by the spectral decomposition

$$
A^{*} A=\sum_{l=1}^{\infty} \sigma_{l}^{2}\left(\Psi_{l}, \cdot\right) \Psi_{l}
$$

of the operator $A^{*} A$, i.e.,

$$
\left(\ln \ldots \ln \left(A^{*} A\right)^{-1}\right)^{-p} v=\sum_{l=1}^{\infty}\left(\ln \ldots \ln \sigma_{l}^{-2}\right)^{-p}\left(\Psi_{l}, v\right) \Psi_{l} .
$$

Examples of elements of the kind of (1.2) for $K=2$ and $p=1$ can be found in inverse problems of diffractive optics (see [3]) and for $K=1$ and for any $p>0$ in satellite gravity gradiometry problem (see [7]).

Without loss of generality we assume that $\|A\| \leqslant M_{K}, M_{K}=m_{K}^{1 / 2}, m_{K}=e^{-1 / m_{K-1}}$, $K \geqslant 2, m_{1}=e^{-1}$, i. e. $\sigma_{l} \leqslant M_{K}, l=1,2, \ldots$

Studies of ill-posed problems are found in [15], where estimates of accuracy for the Tikhonov regularization of equations (1.1) were found with operators of finite and infinite smoothness. Some regularization methods of exponentially ill-posed problems were considered in [9], where, in particular, an algorithm was suggested for solving (1.1) in the case of approximately known operators and the right-hand sides, employing modification of an a posteriori method of choosing a regularization parameter from [19]. In [20] a method was suggested for solving ill-posed problems (1.1) with solutions from (1.2) for $K=1$. It suggests a combination the Tikhonov regularization with Morozov's principle of discrepancy. The indicated combination allows to attain an order-optimal accuracy in the logarithmic scale $O\left(\ln ^{-p}(1 / \delta)\right)$ of recovering solutions from the pointed set for any $p>p_{0}$. Next, article [4] proposes an adaptive strategy of choosing a regularization parameter for general source conditions. Adaptive strategies of choosing a regularization parameter are also discussed in [18], [16]. In [1] the behavior of the regularizing method of equations (1.1) with a solution of the type (1.2) for any $K \in \mathbb{N}$, when the operator $A$ in (1.1) is self-adjoint has been studied. The estimates of the best possible accuracy of recovering the unknown solution from linear ill-posed problems in variable Hilbert spaces are found in [17]. Considered in the same article were projection methods of solving ill-posed problems realizing an order-optimal accuracy.

Remark 1.1. We think it necessary to mention one more method giving an optimal accuracy of solving many classes of ill-posed problems and, in particular, severally ill-posed problems. Namely, on the basis of the method of quasi-solutions [10] the method of extending compact sets was suggested [5]. Later, in [6,21] for the last method the optimal order of accuracy was established on a set of solutions

$$
X_{\rho}=\{x \in X: x=B v, \quad v \in X,\|v\| \leqslant \rho\}
$$

where the quantity $\rho$ is considered to be unknown, and $B$ is an exactly given linear injective compact operator acting from $X$ to $X$.

In connection with the results mentioned in the remark, we should record the principal distinguishing moments of our studies. First, the present article considers a situation opposite to that described above, when in the set of solutions $X_{\rho}$ the operator

$$
B=(\underbrace{\ln \ldots \ln }_{K \text { times }}\left(A^{*} A\right)^{-1})^{-p}
$$


is unknown (because the quantity $p$ is unknown), and the quantity $\rho$ is given exactly. (It should be noted that in the case $B=\left(A^{*} A\right)^{p / 2}$ similar problems were considered, in particular, in $[12,13]$. The algorithms proposed in the above articles allow to determine stably $p$ from the known $\rho$ and to solve problems (1.1) with an order-optimal accuracy on the set (1.3) with $\left.B=\left(A^{*} A\right)^{p / 2}\right)$. Second, here we consider the approximating properties of a concrete combination of the Tikhonov regularization with Morozov's discrepancy. In particular, it will be established that the stated combination is also order optimal on the set of solutions (1.2). Our interest in the described algorithm is explained by the evident simplicity of its realization as compared to several known methods.

Thus, the aim of the present article is to extend the studies of [20] to the case of exponentially ill-posed problems with solutions (1.2) for any $K=1,2, \ldots$ Namely, it will be established that the combination of a discretized Tikhonov regularization with Morozov's discrepancy principle provides the accuracy $O\left((\ln \ldots \ln (1 / \delta))^{-p}\right)$ on the stated class of equations.

\section{A strategy of solving exponentially ill-posed problems}

Any numerical realization of the Tikhonov regularization scheme requires us to carry out all computations with a finite-dimensional approximation $A_{n}$ instead of $A$. In this case, the variation problem $I_{\alpha}(X) \rightarrow$ min is replaced by its finite-dimensional analog

$$
I_{\alpha, n}(x):=\left\|A_{n} x-y_{\delta}\right\|^{2}+\alpha\|x\|^{2} \rightarrow \min ,
$$

where $A_{n}$ is some finite-dimensional approximation with $\operatorname{rank}\left(A_{n}\right)=n$. Then the computation of the approximation $x_{\alpha, n}^{\delta}$ for $x_{0}=A^{-1} y$ requires us to solve the linear operator equation

$$
\alpha x+A_{n}^{*} A_{n} x=A_{n}^{*} y_{\delta} .
$$

Let the finite-dimensional approximation $A_{n}$ be chosen such that

$$
\left\|A-A_{n}\right\| \leqslant \delta \rho^{-1}
$$

Following [19], we shall consider Morozov's discrepancy principle in a form tailored to the finite-dimensional version of the ordinary Tikhonov regularization as a stopping rule.

We will choose the regularization parameter $\alpha$ out of the finite ordered set

$$
\Delta_{q}(\delta)=\left\{\alpha: \alpha=\alpha_{m}:=\alpha_{0} q^{m}, \quad m=0,1, \ldots, \quad \alpha \in\left(\delta^{2}, \alpha_{0}\right)\right\}
$$

for some fixed $q \in(0,1)$. Thus we will compute $x_{\alpha_{m}, n}^{\delta}=\left(\alpha_{m} I+A_{n}^{*} A_{n}\right)^{-1} A_{n}^{*} y_{\delta}$ by solving the equation

$$
\alpha_{m} x+A_{n}^{*} A_{n} x=A_{n}^{*} y_{\delta}, \quad m=0,1,2, \ldots,
$$

until

$$
\left\|A_{n} x_{\alpha_{m}, n}^{\delta}-y_{\delta}\right\| \leqslant d_{0} \delta
$$

with $d_{0} \geqslant \rho / M_{K}+9 / 4$. Here without loss of generality we assume that $\left\|y_{\delta}\right\|>d_{0} \delta$. As we will see in section 4 this strategy ensures the best possible order of accuracy $O\left((\ln \ldots \ln (1 / \delta))^{-p}\right)$ on the source set (1.2) without any information about $p$. 


\section{Auxiliary statements}

Lemma 3.1. Let $\|A\| \leqslant M_{K}$ and $x_{0}=A^{-1} y \in M_{p, \rho}^{\log }(A)$. If $x_{\alpha}=\left(\alpha I+A^{*} A\right)^{-1} A^{*} y$, then for sufficiently small $\alpha$

$$
\left\|A x_{\alpha}-y\right\| \leqslant M_{K}^{-1} \rho \sqrt{\alpha}(\underbrace{\ln \ldots \ln }_{K \text { times }} \frac{1}{\alpha})^{-p} .
$$

Proof. Using the spectral decomposition of the operator $A^{*} A$ we have

$$
\left\|A x_{\alpha}-y\right\|=\left\{\sum_{k=1}^{\infty}\left[\frac{\alpha \sigma_{k}}{\alpha+\sigma_{k}^{2}}\left(\ln \ldots \ln \sigma_{k}^{-2}\right)^{-p}\right]^{2}\left|\left(\Psi_{k}, v\right)\right|^{2}\right\}^{1 / 2},
$$

$\sigma_{k} \in\left(0, M_{K}\right]$. For the following reasoning we need two auxiliary functions: $g_{\alpha}(\lambda)=\lambda \times$ $\left(\alpha+\lambda^{2}\right)^{-1}\left(\ln \ldots \ln \lambda^{-2}\right)^{-p}, \quad \lambda \in\left(0, M_{K}\right]$, and $g(t)=t\left(\ln \ldots \ln t^{2}\right)^{-p}, t \in\left[M_{K}^{-1}, \infty\right)$. First, we will determine the monotony intervals of $g(t)$. Simple calculations show that

$$
g^{\prime}(t)=(\underbrace{\ln \ldots \ln }_{K \text { times }} t^{2})^{-p-1}[\underbrace{\ln \ldots \ln }_{K \text { times }} t^{2}-2 p(\underbrace{\ln \ldots \ln }_{K-1 \text { times }} t^{2})^{-1} \times \ldots \times\left(\ln t^{2}\right)^{-1}] .
$$

$g^{\prime}(t)=0$ is true if and only if

$$
f(t)=2 p
$$

where

$$
f(t)=\underbrace{\ln \ldots \ln }_{K \text { times }} t^{2} \times \underbrace{\ln \ldots \ln }_{K \text { times }} t^{2} \times \ldots \times \ln t^{2} .
$$

The function $f(t)$ is obviously continuous and monotonically increasing on the interval $\left[M_{K}^{-1}, \infty\right)$ with a range $\left[\prod_{l=1}^{K-1} m_{l}^{-1}, \infty\right)$. Therefore, two variants are possible depending on the values of $p>0$ :

a) a root $t_{0}$ of equation (3.2) exists and is unique (if $p \geqslant f\left(M_{K}^{-1}\right) / 2$ ). Then the function $g(t)$ monotonously decreases in $\left[M_{K}^{-1}, t_{0}\right]$ and monotonously increases in $\left[t_{0}, \infty\right)$;

$b$ ) equation (3.2) has no roots (if $p<f\left(M_{K}^{-1} / 2\right)$ ). Then the function $g(t)$ monotonically increases on the interval $\left[M_{K}^{-1}, \infty\right)$.

We prove now that for any $\lambda \in\left(0, M_{K}\right]$ and for sufficiently small $\alpha$

$$
g_{\alpha}(\lambda) \leqslant \frac{M_{K}^{-1}}{\sqrt{\alpha}}(\underbrace{\ln \ldots \ln }_{K \text { times }} \frac{1}{\alpha})^{-p} .
$$

Indeed, if $\lambda<\sqrt{\alpha}$, then $\left(\ln \ldots \ln \left(1 / \lambda^{2}\right)\right)^{-p}<(\ln \ldots \ln (1 / \alpha))^{-p}$ and

$$
g_{\alpha}(\lambda) \leqslant \frac{\lambda}{\alpha}\left(\ln \ldots \ln \frac{1}{\lambda^{2}}\right)^{-p}<\frac{1}{\sqrt{\alpha}}\left(\ln \ldots \ln \frac{1}{\alpha}\right)^{-p} .
$$

Assume now that $\lambda \geqslant \sqrt{\alpha}$. First, consider the case where equation (3.2) has no roots (see $b)$ ). Then, keeping in mind the behavior of $g(t)$ in $\left[M_{K}^{-1}, \infty\right)$, we have

$$
g_{\alpha}(\lambda) \leqslant \frac{1}{\lambda}\left(\ln \ldots \ln \frac{1}{\lambda^{2}}\right)^{-p}=g\left(\frac{1}{\lambda}\right) \leqslant g\left(\frac{1}{\sqrt{\lambda} \alpha}\right)=\frac{1}{\sqrt{\alpha}}\left(\ln \ldots \ln \frac{1}{\alpha}\right)^{-p} .
$$


When a root $t_{0}$ of equation (3.2) exists (see situation a)), by virtue of $t_{0} \leqslant M_{K}$ it follows that $\lambda \in\left[\sqrt{\alpha}, M_{K}\right]$ and $1 / \lambda \in\left[M_{K}^{-1}, 1 / \sqrt{\alpha}\right]$. Then taking into account the behavior of $g(t)$ and the obvious relation $\ln \ldots \ln M_{K}^{-2}=1$, for sufficiently small $\alpha$ we have

$$
\begin{gathered}
g_{\alpha}(\lambda) \leqslant g\left(\frac{1}{\lambda}\right) \leqslant \max \left\{M_{K}^{-1}\left(\ln \ldots \ln M_{K}^{-2}\right)^{-p}, \frac{1}{\sqrt{\alpha}}\left(\ln \ldots \ln \frac{1}{\alpha}\right)^{-p}\right\}= \\
\max \left\{M_{K}^{-1}, \frac{1}{\sqrt{\alpha}}\left(\ln \ldots \ln \frac{1}{\alpha}\right)^{-p}\right\} \leqslant M_{K}^{-1} \frac{1}{\sqrt{\alpha}}\left(\ln \ldots \ln \frac{1}{\alpha}\right)^{-p} .
\end{gathered}
$$

Thus, inequality (3.3) is proved. Now from (3.1) and (3.3) it follows that

$$
\left\|A x_{\alpha}-y\right\|=\alpha\left\{\sum_{k=1}^{\infty}\left[g_{\alpha}\left(\sigma_{k}\right)\left(\Psi_{k}, v\right)\right]^{2}\right\}^{1 / 2} \leqslant M_{K}^{-1} \sqrt{\alpha}\left(\ln \ldots \ln \frac{1}{\alpha}\right)^{-p}\|v\|,
$$

as was to be shown.

Lemma 3.2. Assume that the conditions of Lemma 3.1 are true. Then there exists an $\alpha=\alpha_{k} \in \Delta_{q}(\delta)$ satisfying condition (2.4). Moreover, constants $d_{1}, d_{2}>0$ exist such that

$$
d_{1} \delta \leqslant\left\|A x_{\alpha_{k}}-y\right\| \leqslant d_{2} \delta .
$$

Proof. First, we note that

$$
\left\|x_{0}\right\|=\left\|\left(\ln \ldots \ln \left(A^{*} A\right)^{-1}\right)^{-p} v\right\| \leqslant \rho \sup _{0<\lambda \leqslant m_{K}}\left|\left(\ln \ldots \ln \frac{1}{\lambda^{2}}\right)^{-p}\right| \leqslant \rho .
$$

Moreover, for any bounded operator $B$

$$
\begin{gathered}
B\left(\alpha I+B^{*} B\right)^{-1}=\left(\alpha I+B B^{*}\right)^{-1} B, \quad\left\|\left(\alpha I+B^{*} B\right)^{-1}\right\| \leqslant \alpha^{-1}, \\
\left\|\left(\alpha I+B^{*} B\right)^{-1} B^{*}\right\| \leqslant \frac{1}{2 \sqrt{\alpha}}, \quad\left\|B\left(\alpha I+B^{*} B\right)^{-1} B^{*}\right\| \leqslant 1
\end{gathered}
$$

holds. As in [14], one can represent the residual $A x_{\alpha}-y$ as

$$
A x_{\alpha}-y=A_{n} x_{\alpha, n}^{\delta}-y_{\delta}+S_{1}+S_{2},
$$

where

$$
\begin{gathered}
S_{1}=\left(A_{n}\left(\alpha I+A_{n}^{*} A_{n}\right)^{-1} A_{n}^{*}-I\right)\left(y-y_{\delta}\right)= \\
\left(\alpha I+A_{n} A_{n}^{*}\right)^{-1}\left(A_{n} A_{n}^{*}-\left(\alpha I+A_{n} A_{n}^{*}\right)\right)\left(y-y_{\delta}\right)=\alpha\left(\alpha I+A_{n} A_{n}^{*}\right)^{-1}\left(y-y_{\delta}\right), \\
\left\|S_{1}\right\| \leqslant \alpha\left\|\left(\alpha I+A_{n} A_{n}^{*}\right)^{-1}\right\|\left\|y-y_{\delta}\right\| \leqslant \delta \\
S_{2}=\left(A\left(\alpha I+A^{*} A\right)^{-1} A^{*}-A_{n}\left(\alpha I+A_{n}^{*} A_{n}\right)^{-1} A_{n}^{*}\right) y= \\
\left.\left(A A^{*}\left(\alpha I+A A^{*}\right)^{-1}-\left(\alpha I+A_{n} A_{n}^{*}\right)^{-1} A_{n} A_{n}^{*}\right) y=\alpha\left(\alpha I+A_{n} A_{n}^{*}\right)^{-1}\left(A A^{*}-A_{n} A_{n}^{*}\right)\left(\alpha I+A A^{*}\right)^{-1}\right) y .
\end{gathered}
$$

Now we estimate $S_{2}$ using the representation $\sum_{2}=I_{1}+I_{2}$, where

$$
\begin{gathered}
I_{1}=\alpha\left(\alpha I+A_{n} A_{n}^{*}\right)^{-1}\left(A-A_{n}\right) A^{*}\left(\alpha I+A A^{*}\right)^{-1} A x_{0}, \\
\left\|I_{1}\right\| \leqslant \alpha\left\|\left(\alpha I+A_{n} A_{n}^{*}\right)^{-1}\right\|\left\|A-A_{n}\right\|\left\|A^{*}\left(\alpha I+A A^{*}\right)^{-1} A\right\|\left\|A-A_{n}\right\|\left\|x_{0}\right\| \leqslant \delta, \\
I_{2}=\alpha\left(\alpha I+A_{n} A_{n}^{*}\right)^{-1} A_{n}\left(A^{*}-A_{n}^{*}\right)\left(\alpha I+A A^{*}\right)^{-1} A x_{0},
\end{gathered}
$$




$$
\left\|I_{2}\right\| \leqslant \frac{\left\|A^{*}-A_{n}^{*}\right\|\left\|x_{0}\right\|}{4} \leqslant \frac{\rho}{4}\left\|A-A_{n}\right\| \leqslant \frac{\delta}{4}
$$

Then

$$
\left\|S_{2}\right\| \leqslant \frac{5}{4} \delta
$$

From Lemma 3.1 and (3.5) it follows that

$$
\left\|A_{n} x_{\alpha, n}^{\delta}-y_{\delta}\right\| \leqslant\left\|A x_{\alpha}-y\right\|+\frac{9}{4} \delta \leqslant M_{K}^{-1} \rho \sqrt{\alpha}(\ln \ldots \ln (1 / \alpha))^{-p}+\frac{9}{4} \delta
$$

and, for example, for $\alpha=\delta^{2}(\ln \ldots \ln (1 / \delta))^{2 p}$ we have

$$
\left\|A_{n} x_{\alpha, n}^{\delta}-y_{\delta}\right\| \leqslant M_{K}^{-1} \rho \delta+\frac{9}{4} \delta \leqslant d_{0} \delta .
$$

Taking into account that $\left\|A_{n} x_{\alpha, n}^{\delta}-y_{\delta}\right\|$ monotonically depends on $\alpha$ and, moreover, for sufficiently small $\delta$ and $q>(\ln \ldots \ln (1 / \delta))^{-2 p_{0}}$ the interval $\left(\delta^{2}, \delta^{2}(\ln \ldots \ln (1 / \delta))^{2 p}\right)$ contains at least one element of $\Delta_{q}(\delta)$, we conclude that there exists an $\alpha=\alpha_{k} \in \Delta_{q}(\delta)$ satisfying (2.4). From (3.5) for this $\alpha_{k}$ we have

$$
\left\|A x_{\alpha_{k}}-y\right\| \leqslant\left\|A_{n} x_{\alpha_{k, n}}^{\delta}-y_{\delta}\right\|+\delta+\frac{5}{4} \delta \leqslant d_{0} \delta+\frac{9}{4} \delta=d_{2} \delta .
$$

On the other hand, from (3.1) and (3.5) it follows that

$$
\begin{gathered}
\left\|A x_{\alpha_{k}}-y\right\|=\left\|A x_{q \alpha_{k-1}}-y\right\| \geqslant q\left\|A x_{\alpha_{k-1}}-y\right\| \geqslant \\
q\left[\left\|A_{n} x_{\alpha_{k-1, n}}^{\delta}-y_{\delta}\right\|-\delta-\frac{5}{4} \delta\right] \geqslant q\left[d_{0} \delta-\frac{9}{4} \delta\right]=d_{1} \delta .
\end{gathered}
$$

Thus, we obtain the assertion of Lemma for $d_{2}=d_{0}+9 / 4$ and $d_{1}=q\left(d_{0}-9 \delta / 4\right)$.

Assumption 3.1. Let the function $\varphi:[0, \infty) \rightarrow[0, \infty)$ exists and the operators $A, B$ be such that

1) the function $\varphi(s) / s$ increases in $[0, \infty)$;

2) $\varphi(s)=0$ if and only if $s=0$;

3) $\varphi$ is convex in $[0,1]$;

4) there exists $m>0$ such that

$$
m \int \varphi(1 / \lambda) \lambda d \mu_{x, x}(\lambda) \leqslant\|A x\|^{2}
$$

for all $x \in$ Range $\left(B^{*} B\right)$ where $\mu_{x, x}$ is the spectral measure of $B^{*} B$.

For further discussion we need one result by Mair [15, Theorem 2.10].

Theorem 3.1. Let the conditions of assumption 3.1 holds. If for some $u \in X \quad\|A u\| \leqslant$ $\varepsilon$ and $\|B u\| \leqslant 1$, then

$$
\|u\| \leqslant \sqrt{\varphi^{-1}\left(\varepsilon^{2}\right)}
$$
times.

Let us introduce $\varphi_{K}(s)=s \exp \left(-\exp \ldots \exp s^{-1 /(2 p)}\right), s \in[0, \infty)$, where exp appears $K$

Lemma 3.3. For the function $\varphi_{K}(s)$ conditions 1)-3) of assumption 3.1 hold and

$$
\varphi_{K}^{-1}(s)=(\underbrace{\ln \ldots \ln }_{K \text { times }} \frac{1}{s})^{-2 p}(1+o(1)) .
$$


Proof. The validity of conditions 1), 2) for the function $\varphi_{K}(s)$ is obvious. Let us show now that $\varphi_{K}(s)$ is convex in $[0,1]$, i.e. $\varphi_{K}^{\prime \prime}(s) \geqslant 0$ for any $0 \leqslant s \leqslant 1$.

In the case of $K=1$, this fact was determined earlier (see, for example, [15; 8, Section $3.2])$. Let us carry out an appropriate proof for $K \geqslant 2$. To this end, put $f_{l}(s)=\exp \ldots \exp s$, where exp figures $l$ times and $l=0,1,2, \ldots, K-1$. Here we assume $f_{0}(s)=s$.

It is easy to see that

$$
\varphi_{K}^{\prime \prime}(s)=\frac{1}{(2 p)^{2}} \exp \left(-f_{K-1}\left(s^{-\frac{1}{2 p}}\right)\right) \prod_{j=0}^{K-1} f_{j}\left(s^{-\frac{1}{2 p}}\right) s^{-1}\left[2 p+\prod_{j=0}^{K-1} f_{j}\left(s^{-\frac{1}{2 p}}\right)-\sum_{i=0}^{K-2} \prod_{j=0}^{i} f_{j}\left(s^{-\frac{1}{2 p}}\right)-1\right] .
$$

To reduce computations we put $u=s^{-1 /(2 p)}, u \in[1, \infty)$. Then

$$
\varphi_{K}^{\prime \prime}(u)=\frac{1}{(2 p)^{2}} \exp \left(-f_{K-1}(u)\right) \prod_{j=1}^{K-1} f_{j}(u) u^{2 p+1} r_{K}(u)
$$

where

$$
r_{K}(u)=2 p+\prod_{j=0}^{K-1} f_{j}(u)-\sum_{i=0}^{K-2} \prod_{j=0}^{i} f_{j}(u)-1 .
$$

Since $(2 p)^{-2} \exp \left(-f_{K-1}(u)\right) \prod_{j=1}^{K-1} f_{j}(u) u^{2 p+1}>0$ for any $u>0$, then we have to prove that

$$
r_{K}(u) \geqslant 0
$$

for any $K=2,3, \ldots$ and $u \in[1, \infty)$.

We will carry out the proof of the fact by induction.

First, consider the case where $K=2$. Then

$$
r_{2}(u)=2 p+u \exp (u)-u-1 \geqslant 2 p+e-2>0 .
$$

Assume now that (3.6) holds for $r_{l}(u), \quad l=2, \ldots, K-1$. In other words, we will consider that

$$
2 p+\prod_{j=0}^{l-1} f_{j}(u) \geqslant \sum_{i=0}^{l-2} \prod_{j=0}^{i} f_{j}(u)+1
$$

holds We now prove that (3.6) also holds for $r_{l+1}(u)$. Taking into account inequality (3.7), we have

$$
\begin{aligned}
& r_{l+1}(u):=2 p+\prod_{j=0}^{l} f_{j}(u)-\sum_{i=0}^{l-1} \prod_{j=0}^{i} f_{j}(u)-1=2 p+\prod_{j=0}^{l} f_{j}(u)-\prod_{j=0}^{l-1} f_{j}(u)-\left[\sum_{i=0}^{l-2} \prod_{j=0}^{i} f_{j}(u)+1\right] \geqslant \\
& 2 p+\prod_{j=0}^{l} f_{j}(u)-\prod_{j=0}^{l-1} f_{j}(u)-\left[2 p+\prod_{j=0}^{l-1} f_{j}(u)\right]=\prod_{j=0}^{l} f_{j}(u)-2 \prod_{j=0}^{l-1} f_{j}(u)=\prod_{j=0}^{l-1} f_{j}(u)\left[f_{l}(u)-2\right] \geqslant 0 .
\end{aligned}
$$

The last inequality follows from the evident assertion $e^{s}>2, s \geqslant 1$.

Thus, it has been established that the function $\varphi_{K}(s)$ satisfies condition 3$)$ for any $K \in \mathbb{N}$.

Further let $t=\varphi^{-1}(s)$ hold. Then in view of the definition of the inverse function

$$
s=\varphi(t):=t \exp \left(-f_{K-1}\left(t^{-1 /(2 p)}\right)\right) .
$$


Taking the logarithm of this function $K$ times, we obtain

$$
t=(\ln \ldots \ln (t / s))^{-2 p}
$$

Hence

$$
t=\frac{G_{K}(s)}{(\ln \ldots \ln (1 / s))^{2 p}}
$$

where

$$
G_{K}(s)=\left(z_{K}(s) / \omega_{K}(s)\right)^{2 p}, \quad z_{K}(s)=\underbrace{\ln \ldots \ln }_{K \text { times }} 1 / s, \quad \omega_{K}(s)=\underbrace{\ln \ldots \ln }_{K \text { times }} t / s, \quad t=t(s) .
$$

In article [15], it was established that $G_{1}(s)=1+o(1)$, i.e.,

$$
z_{1}(s)=\omega_{1}(s)(1+o(1)) .
$$

Let us find a logarithmic from this assertion

$$
\ln z_{1}(s)=\ln \omega_{1}(s)+\ln (1+o(1)) .
$$

Since $\ln (1+o(1))=o(1)$, then for $\ln \omega_{1}(s) \neq 0$ we have

$$
G_{2}(s):=\frac{\ln z_{1}(s)}{\ln \omega_{1}(s)}=1+\frac{o(1)}{\ln \omega_{1}(s)} .
$$

As $\omega_{1}(s):=\ln t / s=\ln t^{-\frac{1}{2 p}}($ see [15, Lemma 3.4]), then

$$
\ln \omega_{1}(s)=\ln \frac{t}{s}=-\frac{1}{2 p} \ln t .
$$

Hence, in view of assumption (1.2) $\ln \omega_{1}(s) \rightarrow \infty$ for $s \rightarrow 0$. Then from (3.10) it follows that $G_{2}(s)=1+o(1)$.

Employing the method of induction, we show that

$$
G_{l}(s)=1+o(1) \quad \text { for any } l=3,4, \ldots, K .
$$

Namely, we will consider that the relation $G_{l-1}(s)=1+o(1)$ has already been proved. In other words, we have

$$
\frac{z_{l-1}(s)}{\omega_{l-1}(s)}=1+o(1)
$$

Therefore, $\ln z_{l-1}(s)=\ln \omega_{l-1}(s)+\ln (1+o(1))$. Then for $\omega_{l-1}(s) \neq 1$

$$
G_{l}(s):=\frac{\ln z_{l-1}(s)}{\ln \omega_{l-1}(s)}=1+\frac{o(1)}{\ln \omega_{l-1}(s)} .
$$

From (3.9) it follows that $z_{l-1}(s) \rightarrow \infty$ for $s \rightarrow 0$. Then owing to (3.11) we have $\omega_{l-1}(s) \rightarrow \infty$ for $s \rightarrow 0$, and at the same time $\ln \omega_{l-1}(s) \rightarrow \infty$ for $s \rightarrow 0$. From here by virtue of (3.12) we obtain

$$
G_{l}(s)=1+o(1)
$$

Thus from (3.8) it follows that

$$
\varphi^{-1}(s)=(\ln \ldots \ln 1 / s)^{-2 p}(1+o(1)) .
$$

This completes the proof of the lemma. 
Lemma 3.4. Assume that the conditions of Lemma 3.1 have been satisfied. If $\alpha$ is chosen such that

$$
\left\|A x_{\alpha}-y\right\| \leqslant d_{2} \delta
$$

then

$$
\left\|x_{0}-x_{\alpha}\right\| \leqslant c(\ln \ldots \ln (1 / \delta))^{-p}
$$

when the constant $c$ depends on $d_{2}, p$ and $\rho$.

Proof. We assume $u=\rho^{-1}\left(x_{0}-x_{\alpha}\right)$. Then using the spectral decomposition of $A^{*} A$, we have

$$
u=\rho^{-1} \sum_{k=1}^{\infty} \frac{\alpha}{\alpha+\sigma_{k}^{2}}\left(\ln \ldots \ln \sigma_{k}^{-2}\right)^{-p}\left(\Psi_{k}, v\right) \Psi_{k} .
$$

If

$$
B=\sum_{k=1}^{\infty} \frac{\alpha+\sigma_{k}^{2}}{\alpha}\left(\ln \ldots \ln \sigma_{k}^{-2}\right)^{p}\left(\Psi_{k}, \cdot\right) \Psi_{k},
$$

then it is easy to see that

$$
\|B u\|^{2}=\rho^{-2} \sum_{k=1}^{\infty}\left(\Psi_{k}, v\right)^{2}=\rho^{-2}\|v\|^{2} \leqslant 1
$$

Moreover, for such $B$

$$
\begin{gathered}
\int \varphi_{K}\left(\frac{1}{\lambda}\right) \lambda d \mu_{x, x}(\lambda)=\sum_{k=1}^{\infty} \varphi_{K}\left(\left(\frac{\alpha}{\alpha+\sigma_{k}^{2}}\left(\ln \ldots \ln \sigma_{k}^{-2}\right)^{-p}\right)^{2}\right)\left(\frac{\alpha+\sigma_{k}^{2}}{\alpha}\left(\ln \ldots \ln \sigma_{k}^{-2}\right)^{p}\right)^{2}\left(\Psi_{k}, x\right)^{2}= \\
\sum_{k=1}^{\infty} \exp \left(-\exp \left(\exp \ldots\left(\exp \left(-\left(\frac{\alpha}{\alpha+\sigma_{k}^{2}}\left(\ln \ldots \ln \sigma_{k}^{-2}\right)^{p}\right)^{-1 / p}\right)\right) \ldots\right)\right)\left(\Psi_{k}, x\right)^{2}= \\
\sum_{k=1}^{\infty} \exp \left(-\exp \left(\exp \ldots\left(\exp \left(-\left(\frac{\alpha+\sigma_{k}^{2}}{\alpha}\right)^{1 / p} \ln \ldots \ln \sigma_{k}^{-2}\right)\right) \ldots\right)\right)\left(\Psi_{k}, x\right)^{2} \leqslant \\
\sum_{k=1}^{\infty} \exp \left(-\exp \left(\exp \ldots\left(\exp \left(\ln \ldots \ln \sigma_{k}^{-2}\right)\right) \ldots\right)\right)\left(\Psi_{k}, x\right)^{2}=\|A x\|^{2} .
\end{gathered}
$$

Then from Lemma 3.3 and the above inequality it follows that all conditions of assumption 3.1 are fulfilled for $\varphi_{K}$. In view of (3.13) and the relation

$$
\|A u\|=\rho^{-1}\left\|A x_{0}-A x_{\alpha}\right\|=\rho^{-1}\left\|A x_{\alpha}-y\right\| \leqslant d_{2} \rho^{-1} \delta,
$$

from Theorem 3.1 and Lemma 3.3 it follows that

$$
\|u\|=\rho^{-1}\left\|x_{0}-x_{\alpha}\right\| \leqslant\left(\ln \ldots \ln \left(\frac{\rho^{2}}{d_{2}^{2} \delta^{2}}\right)\right)^{-p}(1+o(1)) \leqslant c\left(\ln \ldots \ln \frac{1}{\delta}\right)^{-p}
$$

which proves the Lemma 3.4. 


\section{The optimal order of accuracy}

Theorem 4.1. Let $\|A\| \leqslant M_{K}$ and $x_{0}=A^{-1} y \in M_{p, \rho}^{\log }(A)$. If $n$ and $\alpha=\alpha_{m} \in \Delta_{q}(\delta)$ have been chosen according to (2.1) and (2.2) respectively, then

$$
\left\|x_{0}-x_{\alpha_{m, n}}^{\delta}\right\| \leqslant c_{0}(\ln \ldots \ln (1 / \delta))^{-p}
$$

where the constant $c_{0}$ depends on $p, \rho, M_{K}, q, d_{0}$.

Proof. First, we note that

$$
\left\|x_{0}-x_{\alpha_{m, n}}^{\delta}\right\| \leqslant\left\|x_{0}-x_{\alpha_{m}}\right\|+\left\|x_{\alpha_{m}}-x_{\alpha_{m, n}}\right\|+\left\|x_{\alpha_{m, n}}-x_{\alpha_{m, n}}^{\delta}\right\|,
$$

where $x_{\alpha_{m, n}}=\left(\alpha_{m} I+A_{n}^{*} A_{n}\right)^{-1} A_{n}^{*} y$. If $\alpha=\alpha_{m}$, then by virtue of Lemma 3.2

$$
d_{1} \delta \leqslant\left\|A x_{\alpha_{m}}-y\right\| \leqslant d_{2} \delta
$$

and from Lemma 3.4 we obtain

$$
\left\|x_{0}-x_{\alpha_{m}}\right\| \leqslant c(\ln \ldots \ln (1 / \delta))^{-p} .
$$

Further, with the help of (3.4) we find

$$
\left\|x_{\alpha_{m, n}}-x_{\alpha_{m, n}}^{\delta}\right\|=\left\|\left(\alpha_{m} I+A_{n}^{*} A_{n}\right)^{-1} A_{n}^{*}\left(y-y_{\delta}\right)\right\| \leqslant \frac{\delta}{2 \sqrt{\alpha_{m}}} .
$$

Moreover,

$$
\begin{gathered}
\left\|x_{\alpha_{m}}-x_{\alpha_{m, n}}\right\|=\left\|\left(\alpha_{m} I+A_{n}^{*} A_{n}\right)^{-1}\left[\alpha_{m}\left(A^{*}-A_{n}^{*}\right)+A_{n}^{*}\left(A_{n}-A\right) A^{*}\right]\left(\alpha_{m} I+A A^{*}\right)^{-1} A x_{0}\right\| \leqslant I_{1}+I_{2}, \\
I_{1}=\alpha_{m}\left\|\left(\alpha_{m} I+A_{n}^{*} A_{n}\right)^{-1}\left(A^{*}-A_{n}^{*}\right)\left(\alpha_{m} I+A A^{*}\right)^{-1} A x_{0}\right\| \leqslant \frac{\left\|A-A_{n}\right\|}{2 \sqrt{\alpha_{m}}} \rho \leqslant \frac{\delta}{2 \sqrt{\alpha_{m}}}, \\
I_{2}=\left\|\left(\alpha_{m} I+A_{n}^{*} A_{n}\right)^{-1} A_{n}^{*}\left(A_{n}-A\right) A^{*}\left(\alpha_{m} I+A A^{*}\right) A x_{0}\right\| \leqslant \frac{\delta}{2 \sqrt{\alpha_{m}}} .
\end{gathered}
$$

Summarizing the estimates we have

$$
\left\|x_{0}-x_{\alpha_{m, n}}^{\delta}\right\| \leqslant c\left(\ln \ldots \ln \frac{1}{\delta}\right)^{-p}+\frac{3}{2} \frac{\delta}{\sqrt{\alpha_{m}}} .
$$

If the parameter choice strategy (2.4) gives $\alpha_{m}$ such that $\alpha_{m}>\delta$, then from (4.2)

$$
\left\|x_{0}-x_{\alpha_{m, n}}^{\delta}\right\| \leqslant c\left(\ln \ldots \ln \frac{1}{\delta}\right)^{-p}+\frac{3}{2} \sqrt{\delta} \leqslant c_{0} \ln ^{-p} \frac{1}{\delta}
$$

follows. On the other hand, if $\alpha_{m} \leqslant \delta$, then Lemma 3.1 and (4.1) lead to the inequality

$$
d_{1} \delta \leqslant\left\|A x_{\alpha_{m}}-y\right\| \leqslant M_{K}^{-1} \rho \sqrt{\alpha_{m}}\left(\ln \ldots \ln \left(1 / \alpha_{m}\right)\right)^{-p} \leqslant M_{K}^{-1} \rho \sqrt{\alpha_{m}}(\ln \ldots \ln (1 / \delta))^{-p} .
$$

It means that

$$
\frac{\delta}{\sqrt{\alpha_{m}}} \leqslant \frac{\rho}{M_{K} d_{1}}\left(\ln \ldots \ln \frac{1}{\delta}\right)^{-p}
$$


Then (4.2) again leads to (4.3). This completes the proof of the theorem.

Let us show now that the quantity $(\ln \ldots \ln (1 / \delta))^{-p}$ gives order optimal accuracy recovering solutions of the kind of (1.2). For that we give the necessary definitions and notation.

The general source condition is denoted in the following way:

$$
M_{\rho}^{\psi}(A):=\left\{x \in X, \quad x=\psi\left(A^{*} A\right) v, \quad\|v\| \leqslant \rho\right\}
$$

where $\psi(t)$ is any continuous increasing function with $\psi(0)=0$. A numerical method $S$ for the approximation $x_{0}$, based on the observations $y_{\delta}$ is given as an arbitrary mapping $S: Y \rightarrow X$. The best possible accuracy of the problem (1.1), (4.4) is defined by minimization over all numerical methods $S$, i.e.,

$$
e\left(M_{\rho}^{\psi}(A), \delta\right):=\inf _{S: Y \rightarrow X} \sup _{x \in M_{\rho}^{\psi}(A)} \sup _{y_{\delta}:\left\|y-y_{\delta}\right\| \leqslant \delta}\left\|x_{0}-S y_{\delta}\right\| .
$$

Let $\Theta(t):=\sqrt{t} \psi(t), \quad t>0$. The following result holds true.

Theorem 4.1 [10]. Suppose that the function $\psi$ from (4.4) such that

1) there is $C<\infty$ for which $\psi(2 t) \leqslant c \psi(t), 0<t \leqslant\|A\|^{2}$;

2) $t \rightarrow \psi^{2}\left(\left(\Theta^{2}\right)^{-1}\right)$ is concave.

Moreover, let the singular numbers $\left\{\sigma_{j}\right\}_{j=1}^{\infty}$ of the operator $A$ in (1.1) obey

3) $\sigma_{j+1}^{2} / \sigma_{j}^{2} \geqslant \gamma>0$.

Then there is $c_{\gamma}>0$ such that

$$
e\left(M_{\rho}^{\psi}(A), \delta\right) \geqslant c_{\gamma} \rho \psi\left(\Theta^{-1}(\delta / \rho)\right), \quad 0<\delta \leqslant \rho\|A\|^{2} .
$$

Note that condition 3) of Theorem 4.1, bounding the rate of decrease in singular values of operator $A$, is satisfied for a wide set of operators, for example, with $\left|\sigma_{l}\right|=l^{-\mu}(\mu>0)$ or $\left|\sigma_{l}\right|=e^{-l}$.

We introduce the notation

$$
\psi_{K}(t)=(\underbrace{\ln \ldots \ln }_{K \text { times }}(1 / t))^{-p} .
$$

Then it is obvious that $M_{p, \rho}^{\log }(A)=M_{\rho}^{\psi_{K}}(A)$ (compare (1.2) and (4.4)). Now we formulate the main result of this article.

Theorem 4.2. Let operator $A$ in (1.1) such that condition 3) of Theorem 3.3 is met. Then the following estimate holds true:

$$
e\left(M_{\rho}^{\psi_{K}}(A), \delta\right)=O\left((\underbrace{\ln \ldots \ln }_{K \text { times }}(1 / \delta))^{-p}\right), \quad 0<\delta \leqslant \min \left\{1 / \rho, \rho\|A\|^{2}\right\} .
$$

The best possible order of accuracy is realized by method (2.1)-(2.4).

Proof. The estimate of the kind $e\left(M_{\rho}^{\psi_{K}}(A), \delta\right) \leqslant c_{0}(\ln \ldots \ln (1 / \delta))^{-p}$ follows from Theorem 3.3 directly. It remains to prove the lower bound for the value of $e\left(M_{\rho}^{\psi_{K}}(A), \delta\right)$. Let us verify the fulfillment of conditions 1), 2) for the function $\psi_{K}(t)$ :

1 ) it is easy to show that for any $K$ the inequality $\psi_{K}(2 t) / \psi_{K}(t) \leqslant c, 0<t \leqslant m_{K}$ holds true.

2) The concavity of the function $t \rightarrow \psi_{K}^{2}\left(\left(\Theta^{2}\right)^{-1}\right)$ is provided by the concavity of the unction $\ln \psi_{K}(t)$ (see. [10, Proposition 1]). The latter fact is proved similarly to Lemma 3.3. 
Then from Theorem 3.3 it follows that

$$
e\left(M_{\rho}^{\psi_{K}}(A), \delta\right) \geqslant c \psi_{K}\left(\Theta^{-1}(\delta / \rho)\right)
$$

where $c=c_{\gamma} \rho, \quad \Theta(t)=\sqrt{t} \psi_{K}(t)$. Thus, to prove the theorem, it is necessary to bound below the quantity

$$
\psi_{K}\left(\Theta^{-1}(\delta / \rho)\right):=\frac{1}{\left(\ln \ldots \ln \left(1 / \Theta^{-1}(\delta / \rho)\right)\right)^{p}} .
$$

Then from the definition of the inverse function follows that if $s=\Theta^{-1}(t)$, then $t=$ $\sqrt{s}(\ln \ldots \ln (1 / s))^{-p}$. Let the transform

$$
s=t^{2}(\underbrace{\ln \ldots \ln }_{K \text { times }}(1 / s))^{2 p}
$$

and bound below the second multiplier on the right-hand side of equality (4.7). Since the logarithmic function is a monotonically increasing function, then this multiplier achieves its minimum for maximum possible value $s$, i. e., for $s_{0}:=\delta / \rho \geqslant m_{K}$. It is easy to see that

$$
\underbrace{\ln \ldots \ln }_{K \text { times }} \frac{1}{s_{0}}=1 \text {. }
$$

Thus from (4.7) we obtain $1 / s \leqslant 1 / t^{2}$. Substituting the latter inequality into (4.7), we find that

$$
s \leqslant t^{2}(\underbrace{\ln \ldots \ln }_{K \text { times }}\left(1 / t^{2}\right))^{2 p} .
$$

As a result, we have

$$
t^{2} \leqslant s:=\Theta^{-1}(t) \leqslant t^{2}\left(\ln \ldots \ln \left(1 / t^{2}\right)\right)^{2 p} .
$$

The combination of (4.6) and (4.8) gives the estimate

$$
\psi_{K}\left(\Theta^{-1}(\delta / \rho)\right) \geqslant \frac{1}{\left(\ln \ldots \ln (\delta / \rho)^{2}\right)^{p}} .
$$

From the condition $\delta / \rho \leqslant m_{K}$ it follows that

$$
\ln \ldots \ln (\rho / \delta)^{2} \leqslant 2 \ln \ldots \ln (\rho / \delta) .
$$

Then

$$
\psi_{K}\left(\Theta^{-1}(\delta / \rho)\right) \geqslant \frac{1}{2^{p}(\ln \ldots \ln (\rho / \delta))^{p}} \geqslant \frac{1}{4^{p}(\ln \ldots \ln (1 / \delta))^{p}},
$$

which by virtue of (4.5) completes the proof of the theorem.

Remark 4.1. Let us carry out a comparative analysis of the proposed method (2.1)(2.4) with the previously known methods that are optimal on the set of solutions (4.4). In [2], on the basis of the ordinary Tikhonov regularization an algorithm has been constructed such that its optimality has been established only in the case of an a priori choice of the regularization parameter. It is obvious that the situation considered in the present article is of a wider character in view of the fact that within the framework of method (2.1)-(2.4) the parameter $\alpha$ is choosen a posteriori. Moreover, in $[16,18]$ on the basis of projective discretization some (order) optimal strategies for solving problems (1.1), (4.6) were constructed. An advantage of our method is the possibility of variation of arbitrary discretization schemes within the framework of condition (2.1). 


\section{References}

1. A. B. Bakushinski, M. Yu. Kokurin, Iterative methods for solution of ill-posed operator equations with smooth operators (Russian), Moscow, Editorial URSS, 2002.

2. A. Böttcher, B. Hofmann, U. Hofmann, U. Tautenhanhn, and M. Yamamoto, Convergence rates for Tikhonov regularization from different kinds of smothness conditions, Applicable Analysis, 85 (2006), no. 5, pp. $555-578$.

3. G. Bruckner, J. Cheng, and M. Yamamoto, An inverse problem in diffractive optics: conditional stability, Inverse Problems, 18 (2002), pp. 415-433.

4. H. Cao and S. Pereverzev, Natural linearization for the identification of a diffusion coefficient in a quasi-linear parabolic system from short-time observations, Inverse problems, 22 (2006), pp. 2311-2330.

5. I. N. Dombrovskaya and V.K. Ivanov, Some questions to the theory of linear equations in abstract spaces (Russian), Sibirskii Mat. Zhurnal, 16 (1965), pp. 499-508.

6. K. Y. Dorofeev, N. N. Nikolaeva, V. N. Titarenko, and A. G. Yagola, New approaches to error estimation to ill-posed problems with applications to inverse problems of heat conductivity, J. of Inverse and Ill-posed Problems, 10 (2002), no. 2, pp. 155-170.

7. W. Freeden and F. Schneider, Regularization wavelets and multiresolution, Inverse Problems, 14 (1998), pp. 225-243.

8. T. Hohage, Iterative methods in inverse obstacle scattering: regularization theory of linear and nonlinear of exponentially ill-posed problems, Doctoral thesis, Linz, 1999.

9. T. Hohage, Regularization of exponentially ill-posed problems, Numer. Funct. Anal. Optim., 21 (2000), pp. 439-464.

10. V. K. Ivanov, V.V.Vasin, and V.P. Tanana, The theory of linear ill-posed problems and its applications (Russian), Nauka, Moskow, 1978, p. 206.

11. M. Yu. Kokurin, and N. A. Yusupova, On necessary and sufficient conditions for the slow convergence of methods for solving linear ill-posed problems (Russian), Izv. Vyssh. Uchebn. Zaved. Mat. (2002), no.2, pp. 81-84.

12. A. S. Leonov and A. G. Yagola, Adaptive regularizing algorithms for the solution of ill-posed problems (Russian), Vestnik Moskov. Univ. Ser.3. Fiz. Astronom, (1998), no. 2, pp. 62-63.

13. A. S. Leonov and A. G. Yagola, Optimal methods for the solution of ill-posed problems with sourcewise represented solutions (Russian), Fundam. Prikl. Mat. 4 (1998), no. 3, pp. 1029-1046.

14. P. Maas and A.Rieder, Wavelet-accelerated Tikhonov-Phillips regularization with applications, in Inverse Problems in medical imaging and nondestructive testing, ed. H.W. Engl, Springer, Wien, 1997, pp. 134-158.

15. B. A. Mair, Tikhonov regularization for finitely and infinitely smoothing operators, SIAM J. Math. Anal., 25 (1994), pp. 135-147.

16. P. Mathe and S. Pereverzev, Discretization strategy for linear ill-posed problems in variable Hilbert scales, Inverse Problems, 19 (2003), no. 6, pp. 1263-1277.

17. P. Mathe and S. Pereverzev, Geometry of linear ill-posed problems in variable Hilbert spaces, Inverse Problems, 19 (2003), no. 3, pp. 623-631.

18. P. Mathe and S. Pereverzev, The discretized discrepancy principle under general source conditions, J. Complexity, 22 (2006), no. 3, pp. 371-381.

19. R. Plato and G. Vainikko, On the regularization of projection methods for solving ill-posed problems, Numer. Math., 57 (1990), pp. 63-79.

20. E. Schock and S. V.Pereverzev, Morozov's discrepancy principle for Tikhonov regularization of severely ill-posed problems in finite-dimensional subspaces, Numer. Funct. Anal. Optim., 21 (2000), pp. 901-916.

21. A. G. Yagola and K. U. Dorofeev, Sourewise sets and a posteriori error estimates for ill-posed problems, in "A. G. Ramm, P. N. Shivakumar and A. V. Strauss (Eds.), Fields Institute Communications: Operator Theory and Its Applications", Providence, RI: American Mathematical Society, 25 (2000), pp. 543-550. 